\title{
Large-Scale Extended Emission Around the Helix Nebula
}

\author{
A. K. Speck, M. Meixner, D. Fong, P. R. McCullough, D. E. Moser \& T. \\ Ueta
}

Astronomy Dept., University of Illinois at Urbana/Champaign, IL 61801 .

We have presented new observations of the ionized gas, molecular gas and cool dust in the Helix nebula (NGC 7293). The ionized gas is observed in the form of a $\mathrm{H} \alpha$ image, which is constructed using images from the Southern H-Alpha Sky Survey Atlas (SHASSA). The molecular emission was mapped using the $\mathrm{H}_{2} v=1 \rightarrow 0 \mathrm{~s}(1)$ line at $2.122 \mu \mathrm{m}$. The far-infrared (FIR) observations were obtained using ISOPHOT on the Infrared Space Observatory (ISO).

The $\mathrm{H} \alpha$ observations are more sensitive than previous measurements and show that the nebula is elongated in the east-west direction to a radius of $\sim 1200^{\prime \prime}$ which equates to a spatial radial extent of more than a parsec (assuming a distance to the Helix of $\sim 200 \mathrm{pc}$ ). The $\mathrm{H} \alpha$ emission exhibits point-symmetric structures in the NE-SW, E-W, and SE-NW directions.

High-resolution $\mathrm{H}_{2}$ observations show that the molecular emission is confined to that part of the nebula seen in the classic optical image. Furthermore, comparison of the $\mathrm{H}_{2}$ emission strength with time-dependent models for photo-dissociation regions (PDRs) shows that the emission arises from thermal excitation of the hydrogen molecules in PDRs and not from shocks.

The FIR images, at 90 and $160 \mu \mathrm{m}$, are mostly due to thermal emission from cool dust grains, but include a small contribution from ionized atomic lines. The FIR emission extends to $\sim 1100^{\prime \prime}$ radius, which equates to a spatial radial extent of more than a parsec.

Assuming that the outer layers of the circumstellar shell have spherical symmetry, radiative transfer modeling of the emission in $\mathrm{H} \alpha$ gives a shell mass of $\sim 1.5 \mathrm{M}_{\odot}$. However, the modeling does not cover the outer most part of the shell (beyond $\sim 600^{\prime \prime}$ radius) and therefore this is a lower limit for the shell mass. Moreover, the models suggest that either $\sim 80-90 \%$ of the dust mass is in grains larger than $3.5 \mu \mathrm{m}$ or there is much less dust in the Helix than previously thought. Furthermore, the FIR data suggest that small dust grains close to the central star are destroyed by the high energy photons from the star.

Details of these results are given in Speck et al. 2002, AJ, 123, 346 